\title{
Agrandamiento gingival por amlodipina: Reporte de caso
}

\author{
Gingival enlargement due to Amlodipine: Case report \\ Aumento gengival devido à amlodipina: Relato de caso
}

Recibido: 10/06/2021 | Revisado: 13/06/2021 | Acepto: 14/06/2021 | Publicado: 28/05/2021

Valentina Martínez Peraza

ORDID: https://orcid.org/0000-0002-4033-2590

Universidad de Cuenca, Ecuador

E-mail: valentina.martinez@ucuenca.edu.ec

Katherine Andrea Romero Espinoza

ORCID: https://orcid.org/0000-0002-7843-9676

Universidad de Cuenca, Ecuador

E-mail: andrea.romero@ucuenca.edu.ec

\section{Resumen}

El agrandamiento gingival es el aumento del volumen de la encía mayormente ocasionado por cambios inflamatorios asociados a la acumulación prolongada de biopelícula dental e inducido por fármacos, dentro de los cuales los bloqueadores de calcio como la amlodipina se destacan. La amlodipina es un fármaco de 3era generación, comúnmente usado para tratar condiciones como la hipertensión, arritmias y anginas de pecho. La prevalencia registrada para el agrandamiento gingival inducido por amlodipina, en pacientes con cardiopatías, oscila entre un $3 \%$. El agrandamiento gingival inducido por amlodipina se debe a una interacción entre los fibroblastos gingivales, la bioquímica de los mediadores de la inflamación y los metabolitos del medicamento. A continuación, se presenta el reporte de caso de un hombre de 57 años, hipertenso, con agrandamiento gingival inducido por amlodipina, y con diagnóstico de Periodontitis estadio 3 grado C, en el que se realizó tratamiento de terapia básica periodontal y se sustituyó la amlodipina por otro fármaco antihipertensivo, adicionalmente para la resolución final del caso se realizó gingivectomía y raspado y alisado radicular a cielo abierto en bolsas recidivantes. Se concluye que la sustitución del fármaco inductor del agrandamiento gingival y una adecuada terapia periodontal puede mejorar la salud gingival del paciente y su calidad de vida.

Palabras clave: Amlodipina; Bloqueadores de canales de calcio; Agrandamiento gingival; Periodontitis; Biopelícula dental.

\begin{abstract}
Gingival enlargement is an increase in the size of the gum mostly caused by inflammatory changes associated with the prolonged accumulation of biofilm and induced by drugs, among which calcium channel blockers such as amlodipine stands out. Amlodipine is a 3rd generation drug, commonly used to treat conditions such as hypertension, arrhythmias and angina pectoris. The prevalence recorded for amlodipine-induced gingival enlargement, in patients with heart disease, ranges between 3\%. Gingival enlargement induced by amlodipine is due to an interaction between gingival fibroblasts, the biochemistry of inflammation mediators and drug metabolites. The following is a case report of a 57 -year-old man, hypertensive, with amlodipine-induced gingival enlargement, and with a diagnosis of periodontitis stage 3 grade $\mathrm{C}$, who underwent basic periodontal therapy treatment and amlodipine was replaced by another antihypertensive drug, additionally for the final resolution of the case, a gingivectomy and open flap scaling and root planning in recurrent pockets was performed. It is concluded that the replacement of the drug induced gingival enlargement and adequate periodontal therapy can improve the patient's gingival health and quality of life.
\end{abstract}

Keywords: Amlodipine; Calcium channel blockers; Gingival enlargement; Periodontitis; Biofilm.

\section{Resumo}

O aumento gengival é o aumento do volume gengival, causado principalmente por alterações inflamatórias associadas ao acúmulo prolongado de biofilme e induzidas por drogas, entre as quais se destacam os bloqueadores dos canais de cálcio como a amlodipina. A amlodipina é um medicamento de $3^{\mathrm{a}}$ geração, comumente usados para tratar doenças como hipertensão, arritmias e angina de peito. A prevalência registrada para aumento gengival induzido por amlodipina em pacientes com doença cardíaca varia entre 3\%. O aumento gengival induzido por amlodipina é devido a uma interação entre fibroblastos gengivais, a bioquímica de mediadores inflamatórios e metabólitos de drogas. A seguir, é relatado o caso de um homem de 57 anos, hipertenso, com aumento gengival induzido por Amlodipina, e com diagnóstico de Periodontite estádio 3 grau $\mathrm{C}$, no qual foi realizado tratamento com terapia periodontal básica e amlodipina foi substituída por outro anti- 
hipertensivo além disso, para a resolução final do caso, foi realizada gengivectomia e a raspagem e alisamento radicular em campo aberto nas bolsas recorrentes. Conclui-se que a reposição do fármaco que induz aumento gengival e adequada terapia periodontal pode melhorar a saúde gengival e a qualidade de vida do paciente.

Palavras-chaves: Amlodipina; Bloqueadores dos canais de cálcio; Aumento gengival; Periodontite; Biofilme.

\section{Introducción}

El agrandamiento gingival es definido como el aumento del volumen de la encía como consecuencia de varias condiciones locales y sistémicas; mayormente se debe a cambios inflamatorios inducidos por la acumulación prolongada de biofilm ( en la mayoría de los casos su remoción induce la resolución del cuadro inflamatorio). No obstante, su aparición se atribuye a factores muy variados: idiopáticos, congénitos, hormonales, neoplásicos, genéticos y farmacológicos. (Bondon-Guitton, Bagheri, Montastruc, \& Centers, 2012; Padrón Chacón, 2014)

Muchas de estas causas son conocidas, pero la más aceptada ha sido el agrandamiento gingival inducido por fármacos como un efecto adverso asociado principalmente a tres clases de medicamentos: anticonvulsivantes (fenitoína), inmunosupresores, (ciclosporina A) , y los bloqueadores de los canales de calcio ( amlodipina, nifedipina, verapamilo, diltiazem). Considerando que la hipertensión arterial es una enfermedad altamente prevalente, y dentro de su terapia farmacológica, los bloqueadores de canales de calcio pueden ser una alternativa terapéutica, se debe analizar la posibilidad de desarrollar agrandamientos gingivales con estos fármacos. (Agrawal, 2015)

Los bloqueadores de canales de calcio actúan reduciendo el paso de los iones de calcio a través de la membrana, debido a la reducción de su permeabilidad afectando la producción de colagenasa. Por consiguiente, ocurre el incremento de la proliferación fibroblástica y la síntesis colágena, dando cabida al agrandamiento gingival. (Banthia, Jain, Banthia, Belludi, \& Jain, 2012)

Sin embargo, es importante considerar que pocos pacientes tratados con fármacos bloqueadores de los canales de calcio van a desarrollar agrandamiento gingival, ya que se sospecha que está asociado a una predisposición genética y susceptibilidad de los fibroblastos a reaccionar en presencia de este tipo de fármacos. Además, los bloqueadores de canales de calcio son metabolizados por la enzima hepática citocromo $\mathrm{P} 450$. El polimorfismo de esta enzima resulta en variaciones interindividuales tanto en la eficacia como en la concentración del fármaco en el plasma y en los tejidos. Esto podría explicar la predisposición en algunos individuos de desarrollar efectos secundarios. (Livada \& Shiloah, 2014)

Es importante mencionar que para que ocurra una respuesta de agrandamiento gingival, además de estar asociada a la exposición del fármaco, es necesaria la existencia de la biopelícula dental, es decir que la presencia de la biopelícula y la inflamación gingival son factores predisponentes para tomar en cuenta en la expresión del agrandamiento gingival inducido por fármacos bloqueadores de los canales de calcio. (Pedro Bullon et al., 1995; P Bullon et al., 1996; Neumann, Willershausen-Zönnchen, Klug, \& Darius, 1996; Seymour, Ellis, \& Thomason, 2000; Tavassoli, Yamalik, Çağlayan, Çağlayan, \& Eratalay, 1998)

Dentro de los bloqueadores de canales de calcio, la amlodipina es un fármaco de 3era generación, que es comúnmente usado para tratar condiciones como la hipertensión, arritmias y anginas de pecho. La prevalencia registrada para el agrandamiento gingival inducido por amlodipina, en pacientes con cardiopatías, oscila entre un 3\%. Esto indica que conocer su mecanismo de acción es de vital importancia, con el afán de prevenir condiciones gingivales que predisponen al desarrollo de enfermedades periodontales y que adicionalmente pueden tener impacto en la condición sistémica del paciente. (Khokhani et al., 1993; Sume et al., 2020; Yolcu \& Aydogdu, 2020)

La amlodipina es el fármaco de elección entre los bloqueadores de canales de calcio, ya que trae consigo menos efectos sistémicos en comparación con los de 1era generación como la nifedipina. Dentro de sus beneficios están los efectos adversos 
reducidos como: hipotensión, edema periférico, náuseas, palpitaciones, síncopes, y es generalmente mejor tolerada. (Khokhani et al., 1993)

El primer caso de agrandamiento gingival asociado con el uso de amlodipina fue publicado en 1994 por Seymour et al. Sin embargo, la mayoría de la evidencia es empírica y los reportes de casos limitados, con pocos estudios de prevalencia en la literatura. (Jorgensen, 1997; Seymour et al., 2000; Seymour, Ellis, Thomason, Monkman, \& Idle, 1994)

De lo reportado se indica que el cuadro clínico inicia con agrandamiento de las papilas interdentales seguido de un incremento en el tejido conectivo, resultando en inflamación gingival. Esto suscita que el agrandamiento gingival inducido por amlodipina se debe a una interacción entre los fibroblastos gingivales, la bioquímica de los mediadores de la inflamación y los metabolitos del medicamento. (Angelopoulos \& Goaz, 1972; Pradhan \& Mishra, 2009)

El mecanismo de acción de la amlodipina aún no es entendido completamente, sin embargo, dos patrones principales inflamatorios y no inflamatorios han sido sugeridos. La hipótesis no inflamatoria hace referencia a que la patogénesis del agrandamiento asociado a la amlodipina está vinculado a la alteración de la acción de la colagenasa como resultado de la disminución del consumo de ácido fólico, mientras que la hipótesis inflamatoria indica que la inflamación puede deberse al efecto tóxico de la amlodipina en bolsas periodontales asociado a los patógenos periodontales que dan cabida a la sobre regulación de citoquinas y a la transformación del factor de crecimiento beta 1 (TGF-B1). Estudios actuales sobre el mecanismo de patogenia del agrandamiento asociado a la amlodipina están direccionados a los efectos directos e indirectos que ésta ocasiona en el metabolismo de los fibroblastos gingivales.(Lafzi, Farahani, \& Shoja, 2006; Lauritano et al., 2019; Srivastava, Kundu, Bandyopadhyay, \& Pal, 2010)

Por lo tanto, el objetivo de este reporte de caso es sugerir la asociación entre los agrandamientos gingivales, la amlodipina, y la biopelícula dental.

\section{Metodología}

El enfoque metodológico del presente artículo consiste en el reporte de un caso clínico, más no en una investigación. La publicación cumple con los principios éticos, puesto que, para llevar a cabo este reporte, el paciente firmó el consentimiento informado de la intervención terapéutica básica periodontal y quirúrgica, así como la aprobación para su publicación; en todo momento se respeta el anonimato del paciente. Para su desarrollo no se requirió la aplicación de encuestas, puesto que se contó con una detallada historia clínica. (Pereira, Shitsuka, Parreira, \& Shitsuka, 2018)

Los autores se autofinancian y tienen el apoyo docente de la Facultad de Odontología de la Universidad de Cuenca.

\subsection{Reporte de caso}

Paciente masculino de 57 años, acudió a consulta de periodoncia por presentar desde hace varios meses, sangrado e inflamación de las encías, con molestias al hablar y al masticar, halitosis, dolor en zonas específicas y movilidad dental a lo que se le adiciona la preocupación por el compromiso estético.

En la anamnesis el paciente manifiesta que es hipertenso controlado, con cardiopatía isquémica previa y recibe tratamiento médico con amlodipina $10 \mathrm{mg}$ diarios desde hace 2 años, adicionalmente fármacos para su condición sistémica.

En la evaluación periodontal clínica y radiográfica se observa presencia de cálculo supra y subgingival, presencia de bolsas periodontales con sondaje promedio de entre 10 - $11 \mathrm{~mm}$, y pérdidas de inserción de entre 5 - $6 \mathrm{~mm}$, movilidad dental grado 1 y 2 en dientes específicos, sangrado al sondaje e inflamación gingival generalizada, así como pérdida de la cresta y pérdidas óseas horizontales y verticales en el análisis radiográfico. Adicionalmente se evidenció supuración en las piezas 43 y 44, dando como 
resultado un diagnóstico de agrandamiento gingival influenciado por fármacos asociado a Periodontitis estadio 3 grado $\mathrm{C}$ generalizada. (Figura 1)

Figura 1. Imagen clínica de la condición periodontal inicial del paciente.

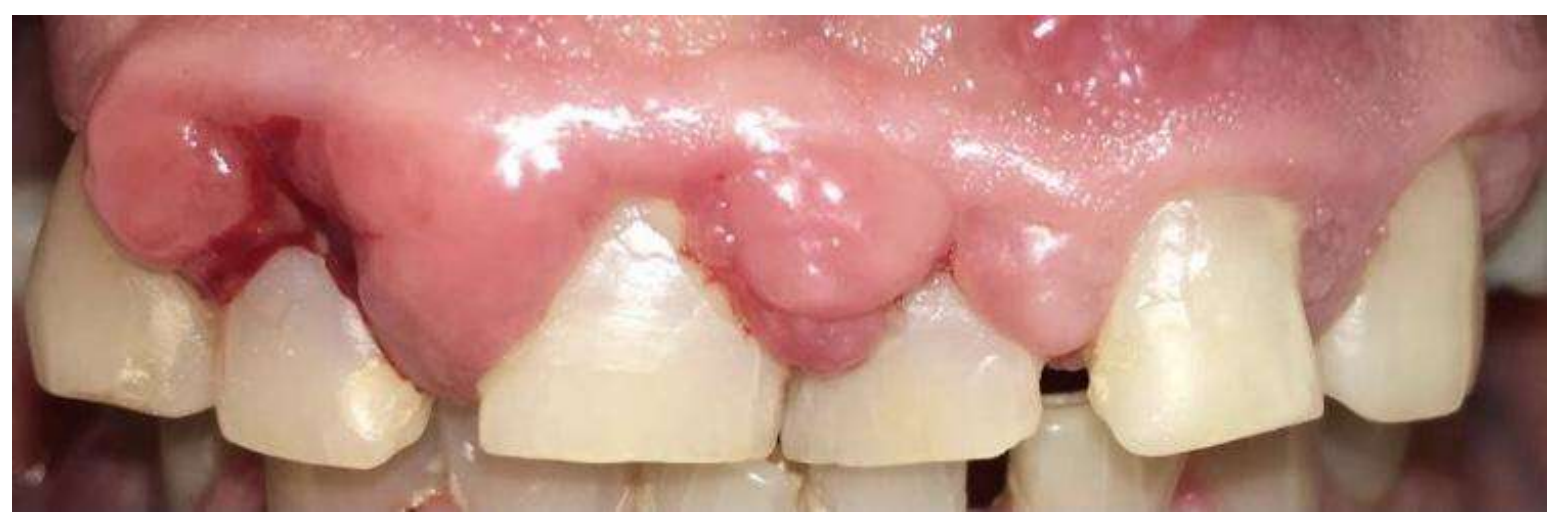

Fuente: Od. Katherine Romero.

Previa atención terapéutica se solicitó interconsulta con el cardiólogo, quien indicó realizar profilaxis antibiótica antes de las diferentes sesiones de raspado y alisado radicular, y con el que se decidió cambiar de fármaco antihipertensivo.

El plan de tratamiento inicial fue la realización de una terapia básica periodontal de raspado y alisado radicular por cuadrante dividida en 4 citas previa profilaxis antibiótica, así como la enseñanza de técnicas de higiene oral con cepillado dental y uso de elementos de higiene interdental.

La terapia con amlodipina fue suspendida y reemplazada por un antagonista de receptor de angiotensina II (Losartán).

Una vez finalizada la fase inicial de la terapia periodontal, se realizó la evaluación de resultados post raspado y alisado radicular, evidenciándose bolsas remanentes en las piezas 13,22,23 y 43, para lo cual se realizó raspado y alisado radicular a cielo abierto con uso de antibióticos locales.

Después de descontinuar la terapia con amlodipina y finalizado el tratamiento periodontal la condición periodontal y el agrandamiento gingival mejoraron notablemente; sin embargo, para restablecer la estética gingival se decidió intervenir quirúrgicamente y realizar una gingivectomía en ambas arcadas. (Figura 2)

Figura 2. Intervención quirúrgica.

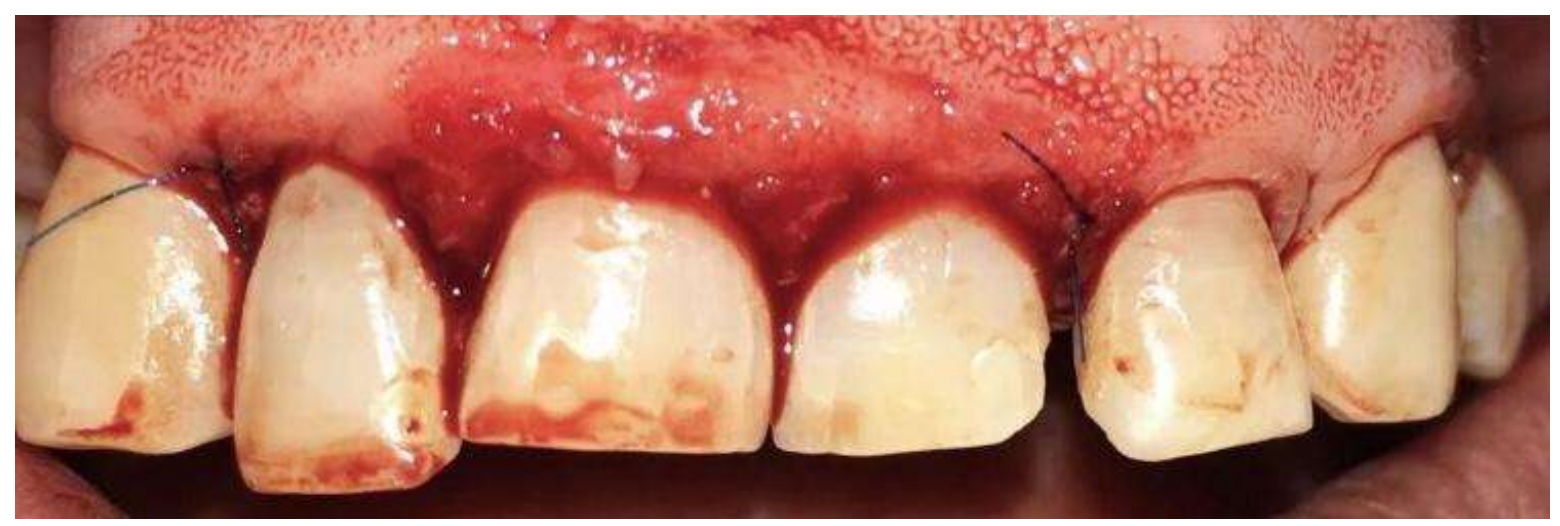

Fuente: Od. Katherine Romero. 
Un mes posterior a la terapia quirúrgica de raspado y alisado radicular y gingivectomía, se realiza un nuevo análisis clínico, determinando cierre total de bolsas, control de la biopelícula dental adecuada, disminución de movilidad dental, y mejoría estética. (Figura 3)

Figura 3. Control clínico postquirúrgico.

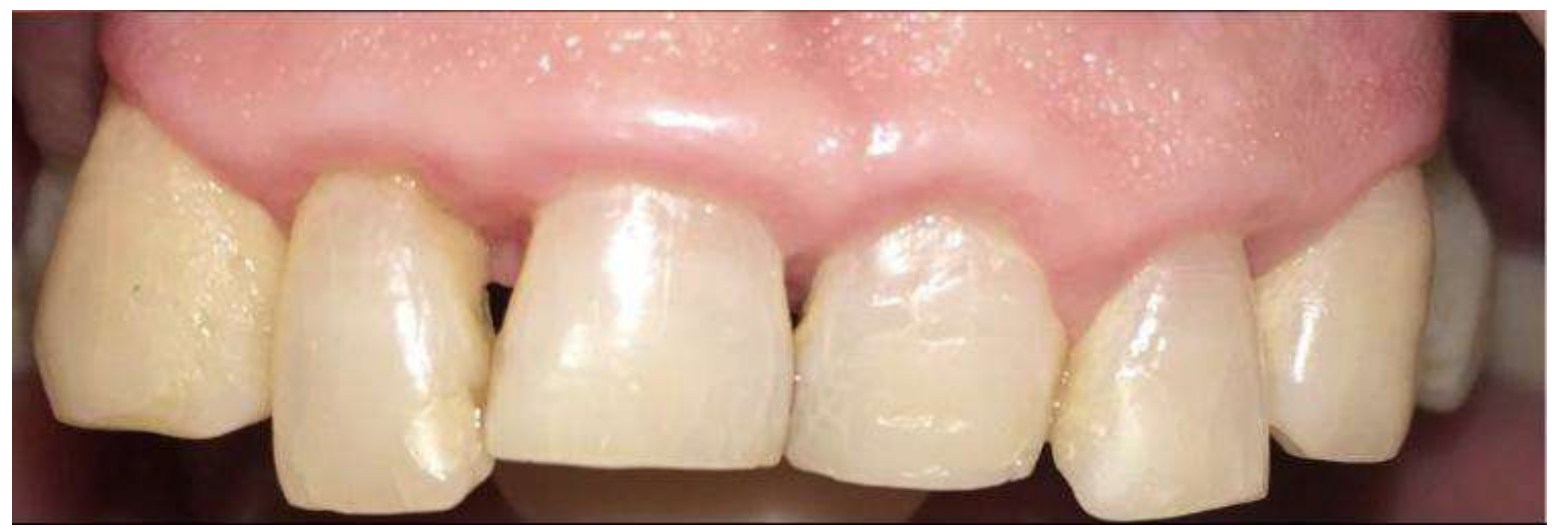

Fuente: Od. Katherine Romero.

Actualmente el paciente asiste a consulta a sus mantenimientos periodontales cada 6 meses; su evolución es satisfactoria y su hipertensión arterial se mantiene controlada.

\section{Resultados y Discusión}

Muchos estudios han reportado la relación que existe entre los fármacos bloqueadores de canales de calcio y el agrandamiento gingival. Entre estos fármacos, la amlodipina es un bloqueador de canal de calcio comúnmente utilizado ya que presenta larga vida media, un alto volumen de distribución, y reducción de efectos adversos en comparación a otros fármacos del mismo grupo. Sin embargo, pocos estudios han investigado la relación entre el tratamiento médico con amlodipina y las alteraciones que ésta puede causar en los tejidos periodontales. (Marshall \& Bartold, 1998; Morisaki et al., 2001; Nishikawa et al., 1991)

Se ha observado que los agrandamientos gingivales aparecen usualmente después de 2 a 15 meses de tratamiento con los fármacos bloqueadores de canales de calcio, y que la amlodipina pareciera ser la responsable de los agrandamientos más severos caracterizándose por ser localizados o generalizados y afectando en su mayoría a los sectores vestibulares anteriores y espacios edéntulos. Los casos reportados hasta el momento evidencian crecimiento de las papilas interdentales y en algunos incluso involucrando el tejido marginal. (Dongari-Bagtzoglou, 2004; Hatahira et al., 2017; Quenel, Keribin, Giran, Tessier, \& Lesclous, 2020)

En este reporte de caso, el paciente tenía tratamiento específico antihipertensivo con amlodipina por indicación del médico cardiólogo, lo que condiciona el estado periodontal por la inducción del agrandamiento gingival favoreciendo el acúmulo de biopelícula dental y por consiguiente la rápida evolución de la enfermedad periodontal. En interconsulta con el médico tratante se decidió sustituir la amlodipina por otra droga antihipertensiva y una vez finalizada la terapia periodontal y la motivación del paciente el cuadro clínico mejoró notablemente.

Se encontró un caso similar reportado por Costa y cols. en Argentina, en el que el agrandamiento gingival asociado al tratamiento médico con amlodipina remitió en un período menor de dos meses, una vez sustituido el fármaco inductor y realizado el 
tratamiento de terapia periodontal, sugiriendo así la asociación entre el empleo de amlodipina y el aumento del volumen gingival. (Costa, Gasparini, \& Valsecia, 2003; Mirabal \& Carbonell, 2016)

Otro estudio a largo plazo reportado por Fardal en pacientes que tomaban bloqueadores de los canales de calcio y recibían tratamiento periodontal concluye que la sustitución del fármaco resultó en mejoría de la salud periodontal de los pacientes y que ésta fue mantenida a largo plazo.(Fardal \& Lygre, 2015)

La patogénesis del agrandamiento gingival inducido por fármacos bloqueadores de canales de calcio no está completamente descifrada. En un artículo de revisión Trackman y Kantarci describieron el agrandamiento gingival como resultado de la acumulación de matriz extracelular y un incremento del número de células. Los fármacos bloqueadores de los canales de calcio y otras drog as promueven la inflamación a través de la liberación de los mediadores inflamatorios por células inmunitarias que activan los fibroblastos y permiten la síntesis de la matriz extracelular. (Trackman \& Kantarci, 2004, 2015)

Adicionalmente, el alto índice de la biopelícula dental resulta en un incremento de inflamación gingival, lo cual actúa como un factor predisponente. Otros estudios reportan que los fármacos bloqueadores de los canales de calcio disminuyen la secreción de las metaloproteinasas - 1 y 3 y colagenasas, resultando en disminución de la degradación de la matriz extracelular. (DongariBagtzoglou, 2004; Livada \& Shiloah, 2014)

Por otra parte, algunos estudios describen la reducción de la apoptosis y el incremento de las células proliferativas, explicando la naturaleza hiperplásica del agrandamiento gingival. (Dongari-Bagtzoglou, 2004; Joshi \& Bansal, 2013; Livada \& Shiloah, 2014)

La terapéutica de los agrandamientos gingivales inducidos por fármacos sugiere iniciar por la eliminación o la sustitución del fármaco en sospecha, siempre en coordinación con el médico tratante. De este modo se podrá corroborar la responsabilidad del fármaco en la inducción del agrandamiento gingival, siempre soportado por una terapia periodontal que incluya motivación y adiestramiento en higiene oral; combinación que devolverá las características clínicas de los tejidos gingivales, recuperando de esto modo la salud bucal.

Sin embargo, son necesarios estudios científicos adicionales que determinen la patogénesis, prevalencia, y caminos terapéuticos adecuados, que sean difundidos tanto en profesionales médicos como odontólogos y conduzcan a la identificación oportuna de los sujetos en riesgo. (Karnik, Bhat, \& Subraya Bhat, 2012; Livada \& Shiloah, 2014)

\section{Conclusiones}

Se concluye que la sustitución del fármaco inductor del agrandamiento gingival y una adecuada terapia periodontal puede mejorar la salud gingival del paciente y su calidad de vida.

La amlodipina podría actuar afectando los tejidos periodontales dando cabida al agrandamiento gingival y agravado por la presencia de la biopelícula dental producto de una deficiente higiene, puntos significativos en esta condición. Podrá ser considerado en el plan de tratamiento periodontal la remoción quirúrgica del agrandamiento gingival cuando ésta así lo demande.

En pacientes hipertensos con antecedente de periodontitis, se recomienda emplear fármacos antihipertensivos diferentes a los bloqueadores de canales de calcio como la amlodipina, ya que la posible hiperplasia gingival puede agravar la enfermedad periodontal de base.

En pacientes hipertensos con manifestación de algún tipo de enfermedad periodontal, es necesario que el odontólogo trabaje como equipo multidisciplinario con el médico tratante, estableciendo medidas terapéuticas coordinadas. 
Se recomienda realizar más estudios clínicos comparativos que analicen el agrandamiento gingival inducido por amlodipina y sus protocolos terapéuticos.

\section{Referencias}

Agrawal, A. A. (2015). Gingival enlargements: Differential diagnosis and review of literature. World Journal of Clinical Cases: WJCC, 3(9), 779.

Angelopoulos, A., \& Goaz, P. (1972). Incidence of diphenylhydantoin gingival hyperplasia. Oral Surgery, Oral Medicine, Oral Pathology, 34(6), 898-906.

Banthia, R., Jain, P., Banthia, P., Belludi, S., \& Jain, A. K. (2012). Amlodipine-induced gingival overgrowth: a case report. The Journal of the Michigan Dental Association, 94(9), 48-51.

Bondon-Guitton, E., Bagheri, H., Montastruc, J. L., \& Centers, F. N. o. R. P. (2012). Drug-induced gingival overgrowth: a study in the F rench P harmacovigilance D atabase. Journal of clinical periodontology, 39(6), 513-518.

Bullon, P., Machuca, G., Angel, M., Rojas, J., Lacalle, J. R., Rios, J. V., \& Velasco, E. (1995). Clinical assessment of gingival size among patients treated with diltiazem. Oral Surgery, Oral Medicine, Oral Pathology, Oral Radiology, and Endodontology, 79(3), 300-304.

Bullon, P., Machuca, G., Martinez-Sahuquillo, A., Rios, J., Velasco, E., Rojas, J., \& Lacalle, J. (1996). Evaluation of gingival and periodontal conditions following causal periodontal treatment in patients treated with nifedipine and diltiazem. Journal of clinical periodontology, 23(7), 649-657.

Costa, S. R., Gasparini, D., \& Valsecia, M. (2003). Hiperplasia gingival fibrosa inducida por fármacos en el NEA. Universidad Nacional Del Nordeste Comunicaciones Científicasy Tecnológicas.

Dongari-Bagtzoglou, A. (2004). Drug-associated gingival enlargement. Journal of periodontology, 75(10), 1424-1431.

Fardal, Ø., \& Lygre, H. (2015). Management of periodontal disease in patients using calcium channel blockers-gingival overgrowth, prescribed medications, treatment responses and added treatment costs. Journal of clinical periodontology, 42(7), 640-646.

Hatahira, H., Abe, J., Hane, Y., Matsui, T., Sasaoka, S., Motooka, Y., \& Ohmori, T. (2017). Drug-induced gingival hyperplasia: a retrospective study using spontaneous reporting system databases. Journal of pharmaceutical health care and sciences, 3(1), 1-11.

Jorgensen, M. G. (1997). Prevalence of amlodipine-related gingival hyperplasia. Journal of periodontology, 68(7), 676-678.

Joshi, S., \& Bansal, S. (2013). A rare case report of amlodipine-induced gingival enlargement and review of its pathogenesis. Case reports in dentistry, 2013.

Karnik, R., Bhat, K. M., \& Subraya Bhat, G. (2012). Prevalence of gingival overgrowth among elderly patients under amlodipine therapy at a large Indian teaching hospital. Gerodontology, 29(3), 209-213.

Khokhani, R., Chopra, P., Karnik, N., Bajan, K., Ailiani, R., Waknis, S., \& Chawla, K. (1993). Amlodipine in mild and moderate hypertension: initial Indian experience. The Journal of the Association of Physicians of India, 41(10), 662-663.

Lafzi, A., Farahani, R. M. Z., \& Shoja, M. A. M. (2006). Amlodipine-induced gingival hyperplasia. Medicina Oral, Patología Oral y Cirugía Bucal (Internet), 11(6), $480-482$.

Lauritano, D., Martinelli, M., Baj, A., Beltramini, G., Candotto, V., Ruggiero, F., \& Palmieri, A. (2019). Drug-induced gingival hyperplasia: An in vitro study using amlodipine and human gingival fibroblasts. International journal of immunopathology and pharmacology, 33, 2058738419827746.

Livada, R., \& Shiloah, J. (2014). Calcium channel blocker-induced gingival enlargement. Journal of human hypertension, 28(1), 10-14.

Marshall, R., \& Bartold, P. (1998). Medication induced gingival overgrowth. Oral diseases, 4(2), 130-151.

Mirabal, Y. Á., \& Carbonell, L. O. M. (2016). Hiperplasia gingival inducida por amlodipino en paciente con periodontitis crónica. Presentación de un caso. MediCiego, $23(1), 36-42$.

Morisaki, I., Dol, S., Ueda, K., Amano, A., Hayashi, M., \& Mihara, J. (2001). Amlodipine-induced gingival overgrowth: periodontal responses to stopping and restarting the drug. Special Care in Dentistry, 21(2), 60-62.

Neumann, C., Willershausen-Zönnchen, B., Klug, C., \& Darius, H. (1996). Clinical assessment of periodontal conditions in patients treated with nifedipine. European journal of medical research, 1(6), 273-279.

Nishikawa, S., Tada, H., Hamasaki, A., Kasahara, S., Kido, J. i., Nagata, T., \& Wakano, Y. (1991). Nifedipine-induced gingival hyperplasia: a clinical and in vitro study. Journal of periodontology, 62(1), 30-35.

Padrón Chacón, R. (2014). Hipertensión arterial. Padrón Chacón R, Almarales Sierra C, Pérez Cárdenas JC, Castellanos Hernández O. Temas de Medicina en Periodoncia. La Habana: Editorial Ciencias Médicas, 69-82.

Pereira, A. S., Shitsuka, D. M., Parreira, F. J., \& Shitsuka, R. (2018). Metodologia da pesquisa científica. 
Research, Society and Development, v. 10, n. 7, e44910717051, 2021

(CC BY 4.0) | ISSN 2525-3409 | DOI: http://dx.doi.org/10.33448/rsd-v10i7.17051

Pradhan, S., \& Mishra, P. (2009). Gingival enlargement in antihypertensive medication. JNMA; journal of the Nepal Medical Association, 48(174), 149-152.

Quenel, L., Keribin, P., Giran, G., Tessier, M.-H., \& Lesclous, P. (2020). Amlodipine-induced gingival enlargement: A case report. Journal of stomatology, oral and maxillofacial surgery, 121(3), 308-311.

Seymour, R., Ellis, J., \& Thomason, J. (2000). Risk factors for drug-induced gingival overgrowth. Journal of Clinical Periodontology: Review article, 27(4), 217223.

Seymour, R., Ellis, J., Thomason, J., Monkman, S., \& Idle, J. (1994). Amlodipine-induced gingival overgrowth. Journal of clinical periodontology, 21 (4), $281-283$.

Srivastava, A. K., Kundu, D., Bandyopadhyay, P., \& Pal, A. K. (2010). Management of amlodipine-induced gingival enlargement: Series of three cases. Journal of Indian Society of Periodontology, 14(4), 279.

Sume, S. S., Berker, E., Ilarslan, Y., Ozer Yucel, O., Tan, C., Goyushov, S., \& Tezcan, I. (2020). Elevated Interleukin-17A expression in amlodipine-induced gingival overgrowth. Journal of periodontal research, 55(5), 613-621.

Tavassoli, S., Yamalik, N., Çağlayan, F., Çağlayan, G., \& Eratalay, K. (1998). The clinical effects of nifedipine on periodontal status. Journal of periodontology, 69(2), 108-112.

Trackman, P., \& Kantarci, A. (2004). Connective tissue metabolism and gingival overgrowth. Critical Reviews in Oral Biology \& Medicine, 15(3), $165-175$.

Trackman, P., \& Kantarci, A. (2015). Molecular and clinical aspects of drug-induced gingival overgrowth. Journal of dental research, 94(4), 540-546.

Yolcu, A., \& Aydogdu, I. (2020). Amlodipine-induced gingival hypertrophy. European Journal of Internal Medicine, 78, 127-128. 